\title{
Location agent: a study using different wireless protocols for indoor localization
}

\author{
Ana Régia de M. Neves ${ }^{1}$, Humphrey C. Fonseca ${ }^{2}$, Célia G. Ralha ${ }^{2}$ \\ ${ }^{1}$ Dept. of Electrical Engineering, University of Brasília, Brazil \\ ${ }^{2}$ Dept. of Computer Science, University of Brasília, Brazil
}

Email address:

regianeves@unb.br (A. R. de M. Neves), humphrey.fonseca@gmail.com (H. C. Fonseca), ghedini@cic.unb.br (C. G. Ralha)

\section{To cite this article:}

Ana Régia de M. Neves, Humphrey C. Fonseca, Célia G. Ralha. Location Agent: A Study Using Different Wireless Protocols for Indoor Localization, International Journal of Wireless Communications and Mobile Computing. Vol. 1, No. 1, 2013, pp. 1-6.

doi: $10.11648 /$ j.wcmc. 20130101.11

\begin{abstract}
Context-aware systems have received greater interest in the computing community. In order to provide relevant services at context-aware applications, the first task is to locate the user, what can be done preferably dynamically and intelligently. However, indoor mobile users localization is not a trivial problem, since it involves checking various devices, transmitting signals simultaneously on the same radio frequency, with possibly the three existing wireless network protocols: Wi-Fi, Bluetooth and ZigBee. In this direction, this paper presents an agent-based architecture with the Location Agent module defined for context-aware applications that uses three artificial neural network algorithms trained for the different protocols: backpropagation, backpropagation with momentum and levenberg-marquardt. Considering the research experimental aspects, a study is presented to compare the neural network algorithms including performance, regression analysis, precision and accuracy. The results indicate that the backpropagation algorithm trained with Bluetooth provides better accuracy (the average error of 0.42 meters) and the backpropagation trained with Wi-Fi provides better precision (73\%). We consider our approach promising since the Location Agent has a quality of service component associated with the neural network algorithms that can choose the best received signal strength to locate indoor users.
\end{abstract}

Keywords: Indoor User Location, Context-Aware Systems, Multiagent System, Quality Of Service, Artificial Neural Network

\section{Introduction}

Mobile devices allow user's mobility and give seamless access to computing resources while moving from one point to another. In this way, there is an increasing interest in context-aware systems that exploit the context to understand various current aspects of users situation to interact with the environment in a more intelligent way [1].

One of the most popular mobile services context aware applications are location based services (LBS) [2]. The LBS are value-added services that use the mobile location to provide relevant information or service to the user at an specific location. Such services can be required outdoor and indoor environments. In outdoor environments LBS are possible due to global positioning system (GPS) that enables accurate positioning. Nowadays, most of mobile devices are equipped with a GPS-receiver. But GPS is not suitable to track mobile users (MU) in indoor environments with acceptable accuracy, since signals might be attenuated by roofs and walls. In this case, the usage of other sensor on mobile devices, such as wireless local area network (WLAN), Bluetooth and ZigBee can be exploited as alternative positioning sensors in indoor environments. At present, indoor positioning remains an open research problem and is our focus of study.

According to [3] existing indoor positioning techniques can be grouped in two main approaches: (i) their level of precision and installation of specialized additional infrastructure such as ultra wide band or ultrasonic which the precision is often high, but are expensive and unsuitable for large scale deployment; and (ii) exploiting already existing network infrastructure, for instance WLAN or inertial sensors for positioning, which the precision is limited, but the system are more economical and can be deployed with few additional expenses. Obviously, wireless network is not designed for the purpose of indoor user localization. However, measurements of the received signal strength (RSS), 
which is founded on the decay law of the received signal versus the distance, imply the location of any MU. On the other hand, suffer from signal attenuation and noise due to hardware characteristics, exacerbated by environmental factors such as walls, furniture and people in motion.

In this research work we focus on methods using existing infrastructures, such as: (i) Wireless fidelity (Wi-Fi) over IEEE 802.11, that includes IEEE $802.11 \mathrm{a} / \mathrm{b} / \mathrm{g}$ standards for WLAN and allows users to access the Internet at broadband speeds when connected to an access point (AP) or in ad hoc mode; (ii) Bluetooth over IEEE 802.15.1, based on a wireless radio system designed or short-range and cheap devices to replace cables for computer peripherals; and (iii) ZigBee over IEEE 802.15.4, defines specifications for low rate wireless personal area network (LR-WPAN) for supporting simple devices that consume minimal power and typically operate in the personal operating space (POS) of $10 \mathrm{~m}$.

According to [4], there are two main groups for indoor location based on wireless network existing infrastructure: (i) signal propagation model and information about the geometry of the building to convert RSS to a distance measurement, with knowledge of the coordinates of the WLAN access points (APs), the method of trilateration can be used to estimate the location of the MU; and (ii) location fingerprinting technique that matches the obtained RSS values with a database containing previously captured RSS patterns in the area of interest. According to [5] location fingerprinting includes two phases: offline and online. In the offline phase, the area of interest is divided into grid points and values of the RSS from multiple APs. The RSS data is collected for a certain period of time and stored in a database, called radio map. During the online phase, the server compares by an algorithm the measured RSS fingerprint to fingerprints stored in the radio map to obtain mobiles location on the grid. The coordinates associated with the fingerprint that provides the smallest distance, for instance Euclidean distance, is returned as the estimated position.

Artificial neural networks (ANN) can also be used to establish a relationship between pattern of RSS samples and location [6]. In [7] an ANN is defined as a massively parallel model, with distributed processors made up of simple processing units called neurons. A variety of ANN models have been proposed and all of them must be trained. Basically, there are two types of training, supervised and unsupervised. Whereas the ANN supervised training knows the desired output, the unsupervised explores correlations between patterns in the data, and organizes into categories from these correlations.

A multilayer feed-forward ANN consists of multiple layers of units connected by directed links and uses supervised training. In this research work there are three supervised learning algorithms being used for training our networks: backpropagation (BP) [8], backpropagation with momentum (BPM) [9] and Levenberg-Marquardt (LM) [10].
The BP algorithm has been widely used as a supervised learning algorithm in feed-forward multilayer ANN based on the Gradient Descent method. That attempts to minimize the error of the network by moving down the gradient of the error curve as stated. However, the BP has a slow convergence. Consequently, many faster algorithms were proposed to speed up the convergence of the BP and can be grouped in two main categories [11]: (i) uses heuristic techniques developed from an analysis of the performance of the standard steepest descent algorithm, for instance BPM, to prevent instabilities caused by a too-high learning rate; and (ii) uses standard numerical optimization techniques, such as LM algorithm, which is an approximation to Newton's method, suitable for training small and medium-sized problems.

According to [12], the use of ANN improved performance and accuracy since they are capable of tackling noisy measurements and are widely used when the correlation between the input and output values of a system is unclear or subject to noise data.

Indoor location accuracy can be measured by the error between the estimated position and the actual position of the mobile device. This characteristic can be improved by Quality of Service (QoS), since it chooses the best RSS to locating users and being one of the key evaluations for LBS. Generally, the QoS is measured in relation to accuracy, response time, availability and consistency [13].

The multi-agent system (MAS) approach is also interesting to apply for indoor localization using together with the ANN and QoS [14]. According to [15], a MAS is characterized by the existence of multiple agents that interact autonomously and work together to solve a problem or to achieve a common goal. In this way, a MAS can be used as an alternative to deal with the complexities of developing an indoor location system, which aggregates multiple wireless protocols. The agents have intrinsic attributes, such as: (i) the ability to perceive and act on the environment; (ii) the possibility of achieving individual goals; (iii) the ease of communicating with other agents; (iv) the ability to perform actions with some level of autonomy; and (v) the ability to provide services. Besides, the MAS architecture can incorporate reactive and deliberative agents that take decisions automatically at execution time.

Our previous work presented and evaluated an agent-based user location module -- the Location Agent Module (LAM), based on fingerprinting technique that uses ANN and QoS in the existing wireless network infrastructure to improve accuracy of indoor user location [14]. Furthermore, we compare results of three ANN supervised learning algorithms (BP, BPM and $L M)$ to indoor localization trained with data of three wireless protocols: Wi-Fi, Bluetooth and ZigBee.

The rest of the paper is organized as follows: in Section 2, we discuss the state of the art related to indoor localization; the LAM architecture is presented in Section 3; Section 4 discuss experimental results, while conclusions and future 
works are presented in Section 5.

\section{State of the Art}

Wireless RSS fingerprinting has become the most promising indoor positioning technique because of its easier deployment and lower cost compare to other methods [5]. In this section some approaches to fingerprint-based indoor localization are going to be presented.

To overcome the limitations suffer from signal attenuation and multipath in Wi-Fi signatures, [16] proposes a new approach to fingerprint based indoor localization that leverages FM broadcast radio signals by achieving localization accuracies similar or better to the one achieved by Wi-Fi signals. Besides augmenting the wireless signature, the SI4735 FM receiver has been used and provides three additional signal quality indicators: signal-to-noise ratio, multipath and frequency offset. Authors show that localization accuracy can be further improved by more than $5 \%$. Moreover, combined FM and Wi-Fi signals to generate wireless fingerprints, the localization accuracy increases as much as $83 \%$ compared to Wi-Fi RSSI used alone as a signature.

Considering the ANN domain, [17] presents a system to find the location of mobile sensor nodes in the harsh, uncertain, dynamic and noisy conditions using some beacon nodes. To achieve this an ANN was developed and validated through some experiments in real world prone to different sources of noise and signal attenuation. The ANN is trained using BMP algorithm and the results are compared with the trilateration technique.

The system presented in [18] combines two different Wi-Fi approaches to user localization: fingerprinting and trilateration using three known AP coordinates detected on the user's device to derive the position. The combination of the two approaches enhances the accuracy of the user position in an indoor environment allowing LBS to be deployed more effectively.

[19] present a software architecture designed for a hybrid location system supporting multiple technologies simultaneously. To demonstrate the application of the architecture and its platform, the paper introduces two case studies based on real deployments: (i) associates ZigBee and Ultra Wide Band mobile nodes, plus the accelerometer; and (ii) uses RSS measurements and a fingerprinting location algorithm.

Another approach is based on collaborative localization of mobile users; for instance, using Bluetooth protocol to improve accuracy and coverage indoors and improve power consumption by duty-cycling GPS outdoors from nearby neighbors [20].

Differing from the presented initiatives, this researchwork focus in the modularization of complexity and interaction of multiple wireless protocols in the indoor environment through the use of an agent-based model. The agent model is appropriate to represent complex interactions among different entities in the indoor environment and permits that decisions are taken automatically at execu- tion time. In addition, the different wireless protocols can be contextualized into different agent types that interact in the environment. Another clear difference in our approach from the cited work [16-20] is the implicit way to deal with the QoS mechanisms, without additional hardware and extra costs.

\section{Architecture Overview}

In [21] previous work, we developed a prototype for indoor user localization using MAS approach. This prototype allowed to define the necessary characteristics for a more complex architecture to context-aware systems. Therefore, we improved our previous architecture with the definition of the LAM, together with the ANN and QoS [14].

Fig. 1 presents LAM composed of three modules and one knowledge base:

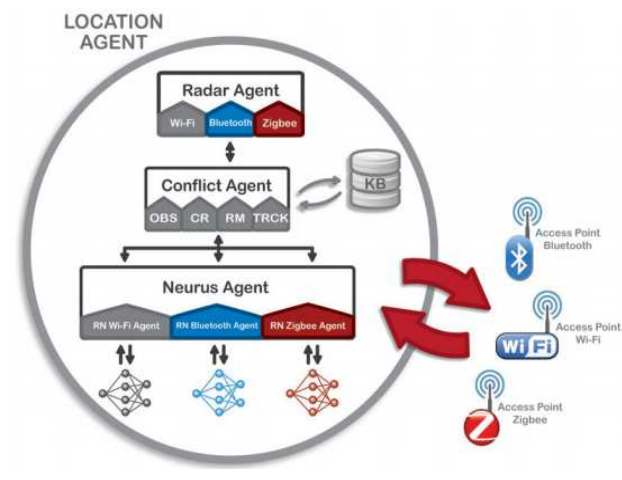

Figure 1. LAM agent-based architecture.

- $\quad$ Radar Agent - it starts the process of indoor users locatlization being composed of three sub-modules: (i) Wi-Fi; (ii) Bluetooth; and, (iii) ZigBee. These sub-modules are responsible for monitoring the environment to gather RSS information and send to the Conflict Agent;

- Conflict Agent - request the RSS for the Radar Agent and is composed of four sub-modules: (i) Observation (OBS), responsible for the RSS request; (ii) Conflict Resolution (CR), responsible to decide which position use, giving access to Knowledge Managed-based; (iii) Knowledge Managed-based $(\mathrm{RM})$, responsible for the inference using If-Then rule statements; and (iv) Tracking (TRCK), responsible for monitoring the user;

- Neurus Agents - receive the RSS of Wi-Fi, ZigBee and Bluetooth infrastructure and transfer them to its respective ANNs, in order to check its own QoS and return location for Conflict Agent.

A prototype was implemented to validate the LAM architecture presented in Fig. 1, which is composed of a set of layers to analyze the map of the RSS signals and the absolute coordinates as illustrate in Fig. 2. Each layer functionality is described in the sequence: 


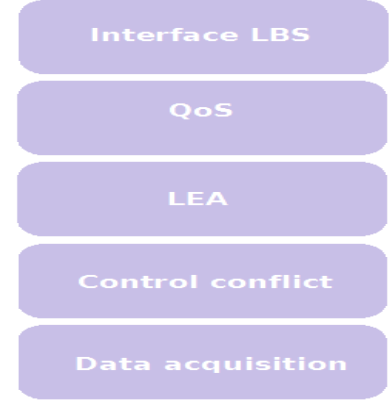

Figure 2. Modular layered architecture.

- $\quad$ LBS interface - an interface that enables communication between the prototype and other applications that make use of the indoor service localization;

- $\quad$ QoS - analyze data from the LEA layer using two levels of QoS predefined: (i) the maximum acceptable error, defined as 1.5 meters according to [11]; and (ii) the signal strength evaluation for assessing the proximity of an access point AP with the acceptable error level defined as 1.0 meters;

- LEA - each independent ANN are responsible to calculate indoor location and the result is obtained from absolute coordinates of users mobile device;

- Control conflict - prepare data obtained at the data acquisition layer and implement two phases: (i) calibration of the account for the hardware differences; and (ii) online to prepare the input values to be used in LEA layer;

- Data acquisition - collect the RSS signals of mobile users in a particular area in the indoor environment.

The LAM agent-based architecture was defined to be a flexible, adaptable and extensible one, since new agents can be added considering other wireless protocols, ANN algorithms and different QoS levels.

\section{Experimental Results and Discussion}

The results presented in [14] show that LAM architecture using different wireless protocols and QoS is suitable for indoor localization process. The use of QoS allows to choose the best signal and leads to more accurate location; also improve the service levels offered. Moreover, as cited in Section I, we compare three ANN algorithms, such as BP, BPM and $L M$. Our experimental tests evaluated the ANN performance, regression analysis, precision and accuracy.

The first experimental test for both ANNs was empirically defined with three types of layers (input, hidden and output) varying the number of neurons at the hidden layer from 100 to 10 . The MLPs are trained using the BP, BPM and LM algorithms. The activation function used for the hidden and output layers is the hyperbolic tangent (tansig). The training parameters were defined with: (i) 10,000 epochs; (ii) target of mean squared error (MSE) equal to zero, since the process of training was intended to be permanent, MSE is the av- erage squared difference between outputs and target; and (iii) the learning rate was set to 0.1 according to the literature [7].

Training automatically stops when generalization stops improving, as indicated by an increase in the MSE. Moreover, regression analysis was performed to measure the correlation between outputs and targets.

In order to calculate the accuracy $(\rho)$, our work uses Euclidean distance that measures the distance between an online RSS value $(\mathrm{X}, \mathrm{Y})$ obtained $(\mathrm{ob})$ and the offline training database RSS $(\mathrm{X}, \mathrm{Y})$ expected (expc) to each point of the grid, as presented in Equation 1.

$$
\rho_{i}=\sqrt{\left(X_{o b t}-X_{\exp e c}\right)^{2}+\left(Y_{\mathrm{obt}}-Y_{\operatorname{expc}}\right)^{2}}
$$

Afterwards, we calculate the value of the average error for the point $\rho$, defined by $\rho_{\mathrm{p}}$, as in Equation 2. Note that, $\mathrm{n}$ represents samples per point in the environment.

$$
\rho_{P}=\frac{1}{\mathrm{n}} \sum_{i p}^{n} \rho_{i}
$$

The accuracy $(\rho)$ is calculated as in Equation 3.

$$
\rho_{P}=\frac{1}{n} \sum_{p p}^{n} \rho_{p}
$$

In order to determine the precision $(\delta)$, the standard deviation of the samples ( $\rho i)$ grouped by the expected point $(\delta p)$

\begin{tabular}{|c|c|c|c|c|}
\hline $\begin{array}{l}\text { ANN algorithms } \\
\text { and Wireless } \\
\text { protocols }\end{array}$ & Performance & Regression $\mathbf{R}$ & $\begin{array}{l}\text { Precision } \\
(\%)\end{array}$ & Accuracy \\
\hline $\begin{array}{l}\text { Backpropagation } \\
\text { Wi-Fi }\end{array}$ & 0,0055 & 0,9859 & 73 & 3,35 \\
\hline $\begin{array}{l}\text { Backpropagation } \\
\text { Bluetooth }\end{array}$ & 0,0068 & 0,985 & 63 & 0,42 \\
\hline $\begin{array}{l}\text { Backpropagation } \\
\text { ZigBee }\end{array}$ & 0,0064 & 0,9844 & 67 & 2,03 \\
\hline $\begin{array}{l}\text { Backpropagation } \\
\text { with } \\
\text { momentum Wi-Fi }\end{array}$ & 0,0059 & 0,9844 & 72 & 0,64 \\
\hline $\begin{array}{l}\text { Backpropagation } \\
\text { with } \\
\text { momentum } \\
\text { Bluetooth }\end{array}$ & 0,085 & 0,9859 & 65 & 2,95 \\
\hline $\begin{array}{l}\text { Backpropagation } \\
\text { with } \\
\text { momentum } \\
\text { ZigBee }\end{array}$ & 0,061 & 0,9595 & 71 & 1,83 \\
\hline $\begin{array}{l}\text { Leven- } \\
\text { berg-Marquardt } \\
\text { Wi-Fi }\end{array}$ & 0,0062 & 0,998 & 69 & 0,89 \\
\hline $\begin{array}{l}\text { Leven- } \\
\text { berg-Marquardt } \\
\text { Bluetooth }\end{array}$ & 0,0064 & 0,995 & 70 & 2,15 \\
\hline $\begin{array}{l}\text { Leven- } \\
\text { berg-Marquardt } \\
\text { ZigBee }\end{array}$ & 0,0095 & 0,886 & 67 & 1,94 \\
\hline
\end{tabular}
is calculated as in Equation 4.

Table 1. ANN algorithms comparison. 


$$
\sigma=\frac{1}{m} \sum_{p p}^{n}\left(1-\frac{\sigma_{p}}{\rho_{p}}\right) \times 100
$$

Table 1 summarizes the main differences among the three ANN algorithms considering performance, regression, precision and accuracy.

For all ANN, the test results to regression analysis presents a stronger level of correlation with a positive increasing value that indicates a good fit. In terms of precision, BP trained with Wi-Fi protocol presents a better result: 73\%, followed by $72 \%$ to BPM also trained with $\mathrm{Wi}-\mathrm{Fi}$ and $71 \%$ to BPM trained with ZigBee protocol. Considering the accuracy, the average error of 0.42 meters to BP trained with Bluetooh, 0.64 meters to BPM trained with Wi-Fi and LM 0.89 meters to LM trained with Wi-Fi.

The results presented in [14] are based on our best ANN architectures and additional level of QoS that improved accuracy in $17 \%$ to the Wi-Fi, $11 \%$ to the Bluetooth and $21 \%$ to the ZigBee protocols.

\section{Conclusions}

As cited in Section I, the goal of context-aware applications is to perceive the users mobile location and dynamically offer them personalized services. In order to achieve this goal, devices and agents must be integrated and cooperate what is possible through the use of an agent-based approach.

Our previous approach presented and evaluated an agent-based user location module -- the LAM, based on fingerprinting technique that uses $\mathrm{ANN}$ and $\mathrm{QoS}$ in the existing wireless network infrastructure [14]. These experiments were a consequence of our first attempt to develop a prototype for indoor localization using MAS approach [21]. Furthermore, we compared results of three supervised ANN learning algorithms (BP, BPM and LM) to indoor localization, trained with data of three wireless protocols (Wi-Fi, Bluetooth and ZigBee) based on fingerprinting location technique.

Analyzing the experimental results, we can say that even though BP algorithm has a slow convergence, it has better performance in indoor localization than the BPM and the LM algorithms. In addition, the use of QoS together with ANN can improve the accuracy of the results as presented in the previous work [14]. For future work, we intend to study the accuracy improvement possibilities by adding other levels of QoS and integrating them to the ZigBee, $\mathrm{WiFi}$ and Bluetooth protocols. Also using semantic resources, such as ontologies, to characterize different contexts we plan to improve the provision of relevant context-aware services to mobile users.

\section{References}

[1] A. K. Dey, G. D. Abowd and D. Salber, "A conceptual framework and a toolkit for supporting the rapid prototyping of context-aware applications," Human-Computer Interaction, vol. 16, no. 2, pp. 97-166, December 2001.

[2] S. Wang, J. Min, and B. K. Yi, "Location based services for mobiles: technologies and standards," Tutorial for IEEE International Conference on Communication (ICC'08), Beijing, China, May 2008.

[3] V. Q. Bien, R. V. Prasad, and I. Niemegeers, "Handoff in Radio over Fiber Indoor Networks at $60 \mathrm{GHz}$," Journal of Wireless Mobile Networks, Ubiquitous Computing, and Dependable Applications, vol. 1, no. 2/3, pp. 71-82, 2010.

[4] Y. Gu, A. Lo, and I. Niemegeers, "A survey of indoor positioning systems for wireless personal networks," IEEE Communications Surveys \& Tutorials, vol. 11, no. 1, pp. 13-32, First Quarter 2009.

[5] V. Honkavirta, T. Perälä, S. Ali-Löytty, and R. Piché, “A comparative survey of WLAN location fingerprinting methods," Proceedings of the 6th Workshop on Positioning, Navigation and Communication (WPNC'09), pp. 243-251, March 2009.

[6] C. S. Chen, "Artificial Neural Network for Location Estimation in Wireless Communication Systems," Sensors, vol. 12, no. 3, pp. 2798-2817, March 2012.

[7] S. O. Haykin, "Neural Networks and Learning Machines," 3rd ed., Prentice Hall, 2008.

[8] Y. Chauvin, and D. E. Rumelhart (ed.) "Backpropagation: Theory, Architectures, and Applications," 1st ed., Psychology Press, 1995.

[9] V. Phansalkar, and P. S. Sastry, "Analysis of the back-propagation algorithm with momentum," IEEE Transactions on Neural Networks, vol. 5, no. 3, pp. 505-506, May 1994.

[10] M. T. Hagan, and M. B. Menhaj, "Training feed forward networks with the Marquardt algorithm," IEEE Transactions on Neural Networks, vol. 5, no. 6, pp. 989-993, November 1994.

[11] S. Lahmiri, "A comparative study of backpropagation algorithms in financial prediction," International Journal of Computer Science, Engineering and Applications (IJCSEA), vol. 1, no. 4, pp. 15-21, August 2011.

[12] M. Altini, D. Brunelli, E. Farella, and L. Benini, "Bluetooth indoor localization with multiple neural networks," Proceedings of the 5th IEEE International Synposium on Wireless Pervasive Computing (ISWPC'10), pp. 295-300, May 2010.

[13] Y. Chen, J. Qi, Z. Sun, and Q. Ning, "Mining user goals for indoor location-based services with low energy and high QoS," Computational Intelligence, vol. 26, no. 3, pp. 318-336, August 2010.

[14] H. C. Fonseca, A. R. d. M. Neves, and C. G. Ralha, "A user location case study using different wireless protocols," Proceedings of the 9th ACM international symposium on Mobility management and wireless access (MobiWac'11), pp. 143-146, October 31-November 4, 2011.

[15] M. Wooldridge, "Introduction to Multi-Agent Systems," 2nd ed., John Wiley \& Sons, Ltd., 2009. 
[16] Y. Chen, D. Lymberopoulos, J. Liu, and B. Priyantha, "FM-based indoor localization", Proceedings of the 10th International Conference on Mobile Systems, Applications, and Services (MobiSys12), pp. 169-182, June 2012.

[17] M. Gholami, N. Cai, R. W. Brennan, "An artificial neural network approach to the problem of wireless sensor network localization," Robotics and Computer-Integrated Manufacturing, vol. 29, no. 1, pp. 96-109, February 2013.

[18] S. Chan, and G. Sohn, "Indoor localization using Wi-Fi based fingerprinting and trilateration techniques for LBS applications", Proceedings of the 7th International Conference on 3D Geoinformation, pp. 1-5, May 2012.
[19] J. Rodas, V. Barral, and C. J. Escudero, "Architecture for multi-technology real-time location systems," Sensors, vol. 13, no. 2, pp. 2220-2253, February 2013.

[20] A. Barreira, P. Sommer, B. Kusy, and R. Jurdak, "Collaborative localization of mobile users with bluetooth: caching and synchronisation," Special Issue on the 3rd International Workshop on Networks of Cooperating Objects, CONET 2012, vol. 9, no. 3, pp. 29-31, July 2012.

[21] A. R. de M. Neves, L. T. Maia, C. G. Ralha, and R. P. Jacobi, "Prototype for indoor localization basedMon multiAgent System," Proccedings of the International Conference on Intelligent and Advanced Systems (ICIAS'10), pp. 1-4, IEEE Computer Society, June 2010. 